\title{
Lack of effect of melatonin on sexual maturation in female rats*
}

\author{
H. Badawi and M. Wilkinson \\ Departments of Physiology and Biophysics and of Obstetrics and Gynecology, Dalhousie University, \\ Halifax, Nova Scotia, Canada B3H 4 H7
}

\begin{abstract}
Summary. Daily subcutaneous injections of $100 \mu \mathrm{g}$ melatonin given to prepubertal female rats housed in 14L:10D or 12L:12D failed to delay puberty as evidenced by the age at which vaginal opening occurred; neither the Sprague-Dawley nor the Wistar strain rats were responsive to melatonin treatment. Reproductive organ weights (ovaries and uteri) at vaginal opening were unaffected by such treatment. Administration of melatonin through the drinking water in doses of 100,500 or $1000 \mu \mathrm{g} /$ day did not alter the timing of puberty or the reproductive organ weights in rats of the Sprague-Dawley or Long-Evans strains (housed in 12L:12D). Our experimental methods are identical to a previous report and we have no explanation for our failure to reproduce the earlier results.
\end{abstract}

Keywords: melatonin; puberty; vaginal opening; rat

\section{Introduction}

Melatonin is known to have an inhibitory effect on the reproductive axis of mammals (Minneman \& Wurtman, 1975). Daily injections of melatonin to adult hamsters results in gonadal regression and decreased testicular and accessory organ weights in males (Reiter et al., 1976; Tamarkin et al., 1976) and cessation of oestrous cyclicity in females (Tamarkin et al., 1976). However, the role of melatonin in the reproductive function of non-seasonal breeders such as the rat remains relatively obscure. The reproductive system of the adult male rat is unresponsive to melatonin (Lang et al., 1983), but some influence has been noted in adult females: daily injections of melatonin result in some disruption of the oestrous cycle (Chu et al., 1964) while injections given during the "critical period' on the day of pro-oestrus result in the blockade of ovulation (Collu et al., 1971; Ying \& Greep, 1973; Sorrentino, 1975). In addition, Reiter et al. (1980) have demonstrated that removal of the olfactory bulbs induces sensitivity to melatonin in immature female Sprague-Dawley rats.

More dramatic effects have been reported when melatonin treatment begins prepubertally (for reviews see Rivest et al., 1986; Lang, 1986). In female rats, daily melatonin injections result in a delay in vaginal opening, decreased ovarian weight, a lower level of pituitary GnRH receptors and a decreased incidence of oestrus after vaginal opening. Male rats treated similarly between Days 20 and 40 of life have decreased testicular and seminal vesicle weights, lower concentrations of plasma LH, FSH and testosterone and fewer pituitary GnRH receptors, as compared to controls. The influence of melatonin on the sexual maturation of the rat is believed to be via the hypothalamus by affecting GnRH release (Rivest et al., 1986; Lang, 1986).

Common to both the hamster and the rat is the importance of the time of injection with respect to the photoperiod. There appears to be a diurnal pattern in the sensitivity of the reproductive axis

*Reprint requests to Dr M. Wilkinson, Department of Physiology and Biophysics, Tupper Med. Bldg., Dalhousie University, Halifax, Nova Scotia, Canada B3H 4H7. 
to melatonin such that sensitivity is greatest in the late afternoon or shortly before the onset of darkness (Rivest et al., 1986; Lang, 1986). Morning melatonin injections are without effect. Diurnal variations in gonadotrophin release are clearly of importance in the attainment of reproductive competence (MacKinnon et al., 1978; Ojeda et al., 1986). Jacobson \& Wilkinson (1986) have suggested that circadian variations in brain neurotransmitter binding sites (receptors; see also Wirz-Justice, 1987), and in particular hypothalamic opioid receptors, may entrain prepubertal hormone secretion. Since melatonin is now thought to be a timekeeping hormone (Karsch, 1986; Reiter, 1987), we wished to know whether the anti-pubertal effects of melatonin (Rivest et al., 1986; Lang, 1986) might be expressed as a disruption of hypothalamic opioid receptor binding rhythms. Unfortunately we have been unable to replicate the report by Rivest et al. (1985) that daily melatonin injections delay rat vaginal opening. We also include in this paper experiments with different rat strains as well as attempts to use melatonin in the drinking water.

\section{Materials and Methods}

Animals. Female rats were obtained from Canadian Hybrid Farms, Halls Harbour, Nova Scotia (Sprague-Dawley) or from Charles River Canada Ltd, St Constant, Quebec (Wistar and Long-Evans) and were housed 4-6 per cage under controlled temperature $\left(20^{\circ} \mathrm{C} \pm 1\right)$ and controlled lighting (fluorescent $34 \mathrm{~W}$ Cool White; General Electric) of either $14 \mathrm{~h}$ light:10 h dark (14L:10D), lights on at 07:00 h (Exps 1 and 2) or 12L:12D, lights on at 06:00 h (all remaining experiments). Food and water were available ad libitum. Rats arriving in litters were used so that each litter provided rats for treatment and control groups. Rats were always weaned on Day 22 of life. Starting at Day 29 rats were checked daily for vaginal opening. On the day of vaginal opening rats were killed and uteri and ovaries were removed and cleaned of associated fat. Corpora lutea were counted and organs weighed.

Injections. Starting on Day 15 of life (except Exp. 1) melatonin $(100 \mu \mathrm{g})$ was injected subcutaneously every day until vaginal opening. Control rats received the vehicle solution. For Exps I and 2 performed with a 14L:10D schedule, injections were given at $16: 30 \mathrm{~h}(9.5 \mathrm{~h}$ after lights on) while for the other experiments in $12 \mathrm{~L}: 12 \mathrm{D}$, injections were given at $17: 00 \mathrm{~h}(11 \mathrm{~h}$ after lights on). Melatonin was dissolved in saline-ethanol $(9: 1 \mathrm{v} / \mathrm{v} ; 0.9 \% \mathrm{NaCl}$ and $100 \%$ ethanol). Samples of $1-5 \mathrm{mg}$ were stored in plastic vials at $-4^{\circ} \mathrm{C}$ and fresh solutions were made daily immediately before injections. Melatonin was obtained from Sigma Chemical Co. (St Louis, MO, U.S.A.) and from Aldrich Chemical Co., Inc. (Milwaukee, WI, U.S.A.). Animals were treated with melatonin from Sigma, while that from Aldrich was purchased for the purpose of comparing melatonin from the two sources by thin-layer chromatography in order to ensure purity of the treatment drug.

Melatonin administration via drinking water. Melatonin was administered to prepubertal female rats via their drinking water in doses of 100 to $1000 \mu \mathrm{g} / \mathrm{rat} / \mathrm{day}$. On the basis of previously determined water consumption of $25 \mathrm{ml} /$ rat/day, melatonin was dissolved in ethanol and diluted with water acidified to a $\mathrm{pH}$ of 2.5 using concentrated $\mathrm{HCl}$. The use of acidified water eliminated the need to change the drinking bottles daily (Hoag et al., 1965; Tober-Meyer \& Bieniek, 1981). Water bottles were also covered with aluminium foil to prevent any adverse effects of light on the melatonin. Control rats received acidified water with ethanol. All rats receiving melatonin via drinking water were housed under 12L:12D.

Statistical analysis. Group means were compared using Student's $t$ test. Differences were considered significant when a $P$ value of 0.05 or less was obtained.

\section{Results}

\section{Experiments 1 and 2}

Methods. Female Sprague-Dawley (SD) rats were obtained at 21 (Exp. 1) or 9 (Exp. 2) days of age and were housed under 14L:10D. Daily melatonin injections were begun on Day 22 (Exp. 1) or Day 15 (Exp. 2).

Results. Melatonin treatment beginning on Day 22 (Exp. 1) was without effect on the age at which vaginal opening occurred. Treatment starting at Day 15 (Exp. 2) resulted in a significant 1.6day delay in vaginal opening (Fig. 1). Melatonin treatment had no effect on uterine weight in either experiment but rats in Exp. 2 showed a reduction in ovarian weight (Fig. 1) $(27 \cdot 8 \pm 1 \cdot 1$ and $24.4 \pm 0.9 \mathrm{mg} / 100 \mathrm{~g}$ body weight in control and experimental rats, respectively). 

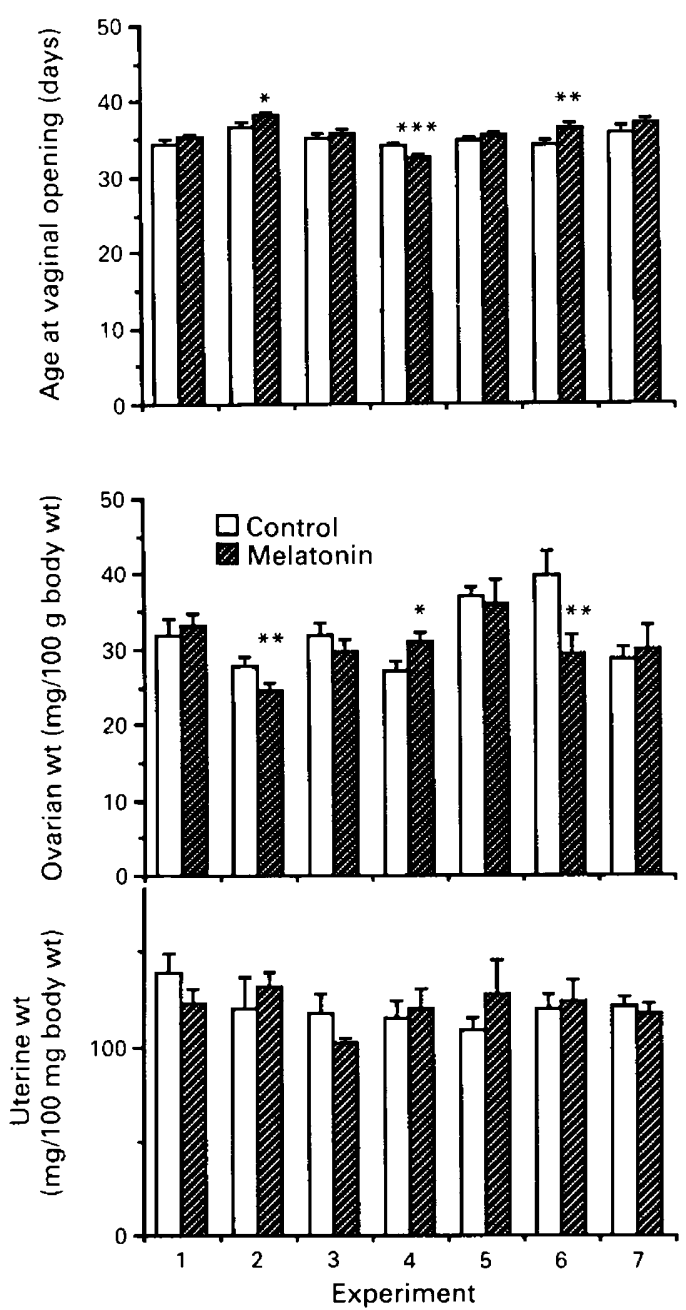

Fig. 1. The age at vaginal opening and ovarian and uterine weights $/ 100 \mathrm{~g}$ body weight (at vaginal opening) of control rats (open bars) and rats treated prepubertally with melatonin (shaded bars) via daily $100 \mu \mathrm{g}$ subcutaneous injections. The conditions of each experiment are detailed in the 'Results' section. Values are mean \pm s.e.m.; $N=7-12$ for control rats and 10-16 for melatonin-treated rats. ${ }^{*} P<0.05,{ }^{* *} P<0.025,{ }^{* * *} P<0.005$.

\section{Experiment 3}

Methods. Female SD rats were received at 9 days of age, housed in 12L:12D and given melatonin injections from Day 15.

Results. There was no delay in vaginal opening in melatonin-treated females and no effect on ovarian or uterine weights (Fig. 1).

\section{Experiment 4}

Methods. SD rats were born at the University Animal Care Center in a photoperiod of 14L: 10D. At 4 days of age female young and their mothers were transferred to 12L:12D. Melatonin was administered by s.c. injections starting on Day 15. 
Results. Vaginal opening was significantly advanced by 1.5 days in rats treated with melatonin (32.5 \pm 0.4 vs $34.0 \pm 0.3$ for controls) (Fig. 1). This was the only incidence of melatonin significantly advancing vaginal opening. It was also the only occasion of melatonin-treated rats having heavier ovaries than controls $(30.8 \pm 1.5$ vs $27.3 \pm 1 \cdot 1 \mathrm{mg} / 100 \mathrm{~g}$ body weight, $P<0.05)$. Uterine weights were similar in both groups.

\section{Experiments 5 and 6}

Methods. The procedures of Exp. 3 were followed except that the rats were of the Wistar strain.

Results. Although the conditions were identical for Exps 5 and 6, melatonin treatment did not show the same effects in both experiments: vaginal opening was delayed in Exp. $6(36.2 \pm 0.6 v s$ $34 \cdot 1 \pm 0.6$ days) but not in Exp. 5. Uterine weight was unaffected in each experiment. Ovarian weight was reduced in Exp. $6(29.3 \pm 2.5 \mathrm{vs} 39.8 \pm 3.1 \mathrm{mg} / 100 \mathrm{~g}$ body weight $)$ and unchanged in Exp. 5.

\section{Experiment 7}

Methods. Following the exact procedures used by Rivest et al. (1985), 12-day pregnant Wistar rats were obtained and the young were born into a photoperiod of 12L:12D. Melatonin injections $(100 \mu \mathrm{g}, \mathrm{s.c}$.$) were begun on Day 15$ of age.

Results. There were no changes in the age at vaginal opening, uterine weight or ovarian weight in melatonin-treated rats compared to controls (Fig. 1).

\section{Experiment 8}

Methods. Female SD rats obtained at 21 days of age were given melatonin in the drinking water at doses of 100 or $500 \mu \mathrm{g} / \mathrm{rat} /$ day.

Results. Neither dose of melatonin affected the day of vaginal opening when compared to controls (Fig. 2). Uterine weight was unaffected by either dose but the dose of $500 \mu \mathrm{g}$ resulted in a reduction of ovarian weight $(29.9 \pm 1.4$ vs $36.9 \pm 2.0 \mathrm{mg} / 100 \mathrm{~g}$ body weight) (Fig. 2).

\section{Experiment 9}

Methods. These were the same as those in Exp. 8 except that the doses of melatonin were increased to 500 and $1000 \mu \mathrm{g} / \mathrm{rat} /$ day and begun on Day 21 .

Results. No effects were observed on vaginal opening, uterine weight or ovarian weight (Fig. 2).

\section{Experiment 10}

Methods. Long-Evans rats were obtained at 21 days of age and given melatonin via the drinking water at doses of 500 or $1000 \mu \mathrm{g} / \mathrm{rat} /$ day, starting on the day of arrival.

Results. A change in vaginal opening was observed only in the rats treated with $500 \mu \mathrm{g}$ melatonin daily (Fig. 2); vaginal opening was advanced by 1.5 days $(32.9 \pm 0.4 v s 34.4 \pm 0 \cdot 8)$. No effects were seen in uterine or ovarian weights in either of the treatment groups (Fig. 2).

\section{Discussion}

Rivest et al. (1985) have reported that daily injections of $100 \mu \mathrm{g}$ melatonin administered $11 \mathrm{~h}$ after the onset of light to female Wistar rats, housed under 12L:12D and starting on Day 15 of life results in a 10-day delay of vaginal opening. After careful consideration of all of these factors and the performance of thin-layer chromatography to ensure purity of the compound, our results failed to show the same effect of melatonin. Since this is unlikely to be due to a strain difference, the most evident explanation seems to be that the sensitivity to melatonin varies between rat colonies. If this is so it weakens the case for the importance of melatonin in the sexual maturation of the female rat. 

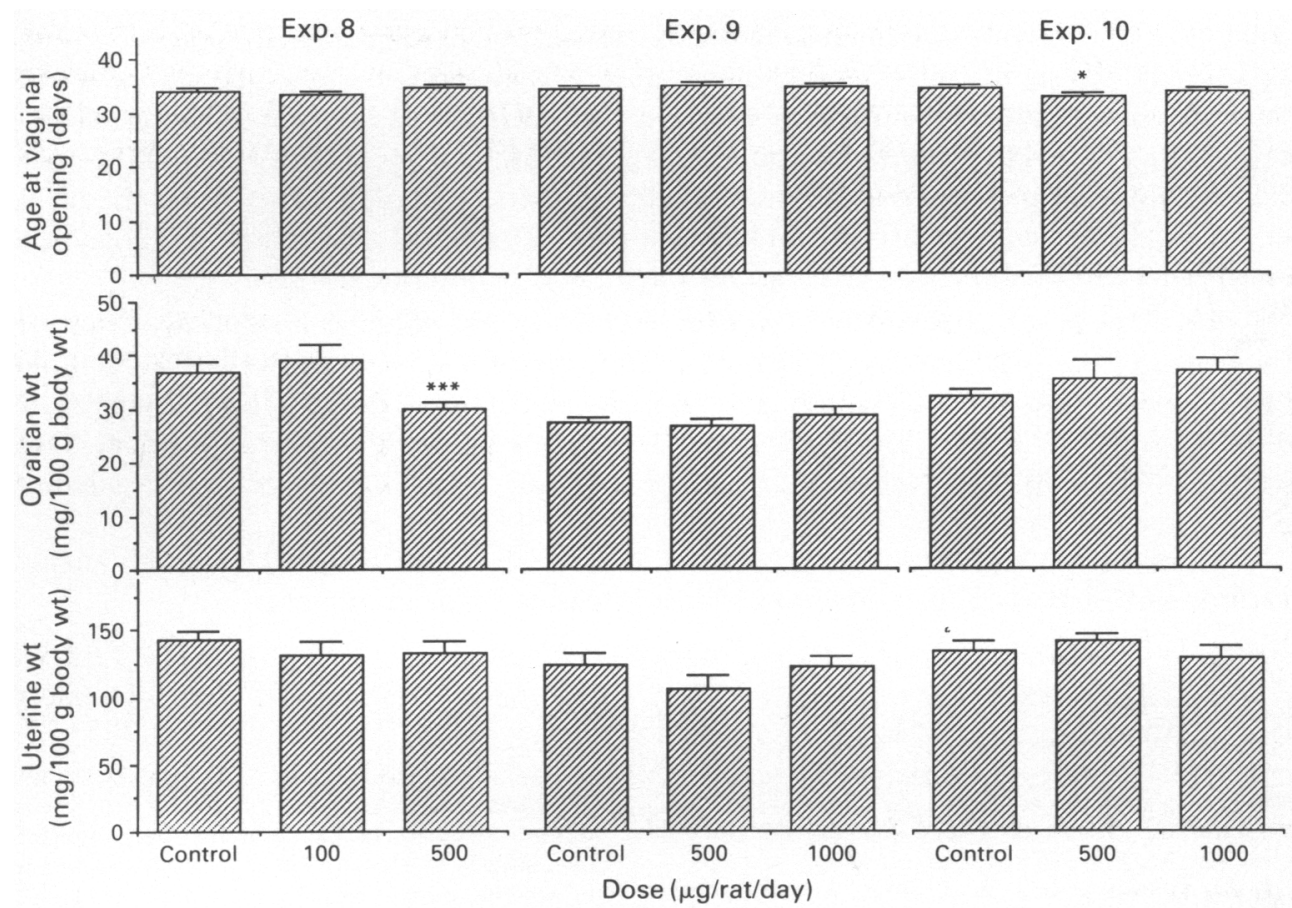

Fig. 2. The effect of administering melatonin through the drinking water on age at vaginal opening and ovarian and uterine weights/100 $\mathrm{g}$ body weight (at vaginal opening). The doses varied from 100 to $1000 \mu \mathrm{g} /$ day. See 'Results' for the conditions of each experiment. Values are mean \pm s.e.m.; $\mathrm{N}=10$ for control rats and $\mathrm{N}=10$ or 12 for melatonin-treated rats. ${ }^{*} P<0.05,{ }^{* * *} P<0.005$.

In our experiments, vaginal opening did occur slightly later in the melatonin-treated rats, but, except in Exp. 4 in which there was a significant advance in the time of vaginal opening, delays in the age at vaginal opening were statistically significant only in Exps 2 and 6 . However, whenever the time of vaginal opening was statistically affected by treatment, the change was never more than $6 \%$ of control values. Modifications of such limited magnitude may be interpreted as merely due to natural variation rather than to a significant physiological effect of melatonin. Nevertheless, when melatonin did alter timing of vaginal opening there was a corresponding and significant change in ovarian weight. Delays in vaginal opening seen in Exps 2 and 6 were accompanied by significant decreases in ovarian weight while the earlier age of vaginal opening in Exp. 4 was associated with increased ovarian weight. Uterine weight was unaffected by melatonin treatment in all experiments. The mean number of corpora lutea per ovary was between 6 and 7 for all experiments and there were no differences between control and melatonin-treated rats.

One can readily see this basic variability in the results of the identical Exps 5 and 6 (Wistar rats). Significant changes in time of vaginal opening and ovarian weight were observed in Exp. 6 but not in Exp. 5. There is no evident explanation for this observation but it again suggests that the observed differences are no more than inherent variation and have no physiologically significant cause. Discrepancies such as these have been reported previously and thus are not surprising. Adams et al. (1965) reported that daily injections of $100 \mu \mathrm{g}$ melatonin between Days 21 and 35 of life delayed vaginal opening in one experiment and not another and Collu et al. (1971) found that ovarian weight was reduced in intraventricular injections of melatonin (starting at age 25 days) in one experiment but not another. Rivest et al. (1985) also commented that time of vaginal opening in some of the rats $(40 \%)$ was unaffected by $100 \mu \mathrm{g}$ melatonin injections (given $9 \mathrm{~h}$ after lights on in 12L:12D). 
Treatment of rats with melatonin in the drinking water effected changes in only two instances: ovarian weight was significantly reduced in Sprague-Dawley rats receiving a daily dose of $500 \mu \mathrm{g}$, and vaginal opening occurred earlier (by $4.4 \%$ ) in Long-Evans rats receiving this same dose. The doses of $100 \mu \mathrm{g}$ and $1000 \mu \mathrm{g}$ produced no effects in any of the experiments. Why $500 \mu \mathrm{g}$ reduced ovarian weight in Exp. 8 but not in Exp. 9 is unclear. Melatonin administered via the drinking water has resulted in gonadal atrophy in male hamsters (Pévet \& Haldar-Misra, 1982), indicating that melatonin can be absorbed unchanged through the gastrointestinal tract.

We can think of no simple explanation to account for our inability to replicate the work of Rivest et al. (1985). The three rat strains available to us appear to be generally unresponsive to melatonin. This suggests that the Swiss colonies used by Rivest et al. (1985) are somehow more sensitive to the anti-pubertal effect of melatonin. It would be useful if these rats could be made available in North America.

This work was made possible by a grant to M.W. from the Canadian MRC. H.B. is indebted to the Dalhousie University Killam Memorial Trust Fund for financial support.

\section{References}

Adams, W.C., Wan, L. \& Sohler, A. (1965) Effect of melatonin on anterior pituitary luteinizing hormone. J. Endocr. 31, 295-296.

Chu, E.W., Wurtman, R.J. \& Axelrod, J. (1964) An inhibitory effect of melatonin on the estrous phase of the estrous cycle of the rodent. Endocrinology 75, 238-242.

Collu, R., Fraschini, F. \& Martini, L. (1971) Blockade of ovulation by melatonin. Experientia 27, 844.

Hoag, W.G., Strout, J. \& Meier, H. (1965) Epidemiological aspects of the control of pseudomonas infection in mouse colonies. Lab. Anim. Care 15, 217-225.

Jacobson, W. \& Wilkinson, M. (1986) Association of diurnal variations in hypothalamic but not cortical

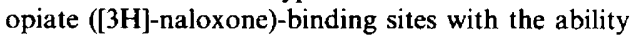
of naloxone to induce $\mathrm{LH}$ release in the prepubertal female rat. Neuroendocrinology 44, 132-135.

Karsch, F.J. (1986) A role for melatonin as a timekeeping hormone in the ewe. J. Neural Transm. 21, 109-124.

Lang, U. (1986) Melatonin and puberty. Pineal Research Reviews 4, 199-243.

Lang, U., Aubert, M.L., Conne, B.S., Bradtke, J.C. \& Sizonenko, P.C. (1983) Influence of exogenous melatonin on melatonin secretion and on the neuroendocrine reproductive axis of intact male rats during sexual maturation. Endocrinology 112, 1578-1584.

MacKinnon, P.C.B., Puig-Duran, E. \& Laynes, R. (1978) Reflections on the attainment of puberty in the rat: have circadian signals a role to play in its onset? $J$. Reprod. Fert. 52, 401-412.

Minneman, K.P. \& Wurtman, R. (1975) Effects of pineal compounds on mammals. Life Sci. 17, 1189-1200.

Ojeda, S.R., Urbanski, H.F. \& Ahmed, C.E. (1986) The onset of female puberty: studies in the rat. Recent Prog. Horm. Res. 42, 385-442.

Pévet, P. \& Haldar-Misra, C. (1982) Effect of orally administered melatonin on reproductive function of the golden hamster. Experientia 38, 1493-1494.

Reiter, R.J. (1987) The melatonin message: duration versus coincidence hypotheses. Life Sci. 40, 2119-2131.

Reiter, R.J., Blask, D.E., Johnson, L.Y., Rudeen, P.K.,
Vaughan, M.K. \& Waring, P.J. (1976) Melatonin inhibition of reproduction in the male hamster: its dependency on time of day of administration and on intact and sympathetically innervated pineal gland. Neuroendocrinology 22, 107-116.

Reiter, R.J., Peterborg, L.J., Trakulrungsi, C. \& Trakulrungsi, W.K. (1980) Surgical removal of the olfactory bulbs increases sensitivity of the reproductive system of female rats to the inhibitory effects of late afternoon melatonin injections. J. exp. Zool. 212, 47-52.

Rivest, R.W., Lang, U., Aubert, M.L. \& Sizonenko, P.C. (1985) Daily administration of melatonin delays rat vaginal opening and disrupts the first estrous cycles: evidence that these effects are synchronized by the onset of light. Endocrinology 116, 779-787.

Rivest, R.W., Aubert, M.L., Lang, U. \& Sizonenko, P.C. (1986) Puberty in the rat: modulation by melatonin and light. J. Neural Transm. 21, 81-108.

Sorrentino, S., Jr (1975) Ovulation in PMS-treated rats with gonadotropin releasing hormone after pentobarbital and melatonin block. Neuroendocrinology 19, 170-176.

Tamarkin, L., Westrom, W.K., Hamill, A.I. \& Goldman, B.D. (1976) Effect of melatonin on the reproductive systems of male and female Syrian hamsters: a diurnal rhythm in sensitivity to melatonin. Endocrinology 99, 1534-1541.

Tober-Meyer, B.K. \& Bieniek, H.J. (1981) Studies on the hygiene of drinking water for laboratory animals. 1 . The effect of various treatments on bacterial contamination. Lab. Anim. 15, 107-110.

Wirz-Justice, A. (1987) Circadian rhythms in mammalian neurotransmitter receptors. Progress in Neurobiology 29, 219-259.

Ying, S. \& Greep, R.O. (1973) Inhibition of ovulation by melatonin by the cyclic rat. Endocrinology $92,333-$ 335 . 